\title{
Influence de l'humidité du sol et de la distribution des racines sur le potentiel hydrique du xylème dans des peuplements de chêne (Quercus sp) de basse altitude *
}

\author{
E Lucot, PM Badot, S Bruckert \\ Université de Franche-Comté, Institut des sciences et techniques de l'environnement, \\ laboratoire de sciences végétales, place Leclerc, 25030 Besançon cedex, France
}

(Reçu le 8 novembre 1993 ; accepté le 4 juillet 1994)

\begin{abstract}
Résumé - Les variations du potentiel hydrique du xylème de rameaux de chênes adultes (Quercus $\mathrm{sp}$ ) ont été mises en relation avec les variations des paramètres climatiques, température et hygrométrie, avec celles des paramètres pédologiques, humidité volumique et polentiel hydrique du sol, et avec les différents types de prospection racinaire. Les mesures ont été effectuées dans 3 stations de l'étage collinéen durant une saison de végétation. Une des stations est établie sur un sol très profond $(4,5 \mathrm{~m})$ ne présentant pas d'obstacle à l'enracinement. La seconde repose sur un sol profond $(3 \mathrm{~m})$ hydromorphe montrant une contrainte à l'enracinement à $50 \mathrm{~cm}$ de profondeur. La dernière présente également une contrainte à $50 \mathrm{~cm}$ et la roche calcaire apparaît à $150 \mathrm{~cm}$. Les calculs de régressions multiples pas à pas montrent que le potentiel du xylème varie au cours de la journée en fonction du potentiel atmosphérique et de l'humidité volumique du sol mesurée entre 40 et $50 \mathrm{~cm}$ et entre 80 et $90 \mathrm{~cm}$ de profondeur. Les relations sont différentes selon la station considérée : plus le sol est contraignant pour l'enracinement, plus le potentiel du xylème est lié aux conditions atmosphériques. Les résultats obtenus montrent que le système racinaire profond assure l'alimentation en eau en conditions de sécheresse. De plus, les 2 espèces de chêne étudiées réagissent au stress hydrique de manière identique.
\end{abstract}

Quercus sp / stress hydrique / eau / sol / potentiel hydrique du xylème / système racinaire

Summary - Influence of soil humidity and roots distribution on xylem water potential in low altitude oak (Quercus sp) planting. The variations in xylem water potential of Quercus $s p$ twigs were studied in comparison with the different types of rooting prospection and changes in air temperature and hygrometry, soil bulk moisture and soil water potential. Measurements were performed in 3 different sites. One of the populations was established on very deep soil $(4.5 \mathrm{~m})$ without any rooting constraint. The second was on hydromorphic deep soil with a rooting constraint at $50 \mathrm{~cm}$ depth. The third also displayed a constraint at $50 \mathrm{~cm}$ and calcareous rocks appeared at $150 \mathrm{~cm}$. Stepwise multiple regressions show

* Cet article est dédié à la mémoire du professeur S Bruckert, prématurément disparu pendant la réalisation de ce travail. 
that the xylem potential fluctuates during the day in accordance with the atmospheric potential and soil bulk moisture, measured between 40 and $50 \mathrm{~cm}$ and 80 and $90 \mathrm{~cm}$ depth. The relationships were different according to the station: the more the soil is constrained for rooting, the more the xylem potential is related to atmospheric conditions. Results show that deep rooting is accountable for water supply during dry conditions. The 2 oak species and their hybrids react identically at water stress.

\section{Quercus sp / water stress / water / soil / xylem water potential / rooting}

\section{INTRODUCTION}

Les échanges d'eau qui s'effectuent à travers le continuum sol-plante-atmosphère régissent pour une large part le fonctionnement des écosystèmes forestiers. Les déficits hydriques, en particulier ceux observés ces dernières années, ont des conséquences non seulement sur la production de bois, mais aussi sur la vitalité des peuplements (Aussenac et Finkelstein, 1983; Aussenac et Levy, 1983 ; Guillaumin et al, 1983 ; Aussenac et Granier, 1984 ; Saugier et al, 1985 ; Badot et Garrec, 1990).

Il est donc important de bien comprendre l'économie de l'eau dans les différents compartiments considérés. Une telle connaissance est susceptible d'apporter une aide à la décision pour la gestion forestière et le reboisement.

L'étude présentée ici a pour objectif de quantifier et d'expliquer les variations du potentiel hydrique du xylème dans des peuplements de chênes adultes établis sur 3 stations présentant des sols très différents. Les variations du potentiel hydrique ont été mises en relation avec le régime hydrique et l'humidité du sol, avec les paramètres racinaires et avec les conditions amosphériques.

\section{MATÉRIEL ET MÉTHODES}

\section{Situation et caractéristiques des stations d'étude}

Les études ont été menées sur 3 stations situées à proximité de Besançon (Doubs, France) vers
$300 \mathrm{~m}$ d'altitude, sur les premiers reliefs de la chaîne jurassienne. Les coordonnées Lambert $x$ et y des 3 stations : Roche-lez-Beaupré, Chevroz et Fontain, sont respectivement 884,75 et 260,75 , 876,75 et $265,20,879,30$ et 249,10 . Le climat régional est de type tempéré avec $1000 \mathrm{~mm}$ de précipitations moyennes par an, $10^{\circ} \mathrm{C}$ de température moyenne annuelle et un déficit hydrique en été. Les sites de Fontain ( $355 \mathrm{~m}$ d'altitude) et de Roche-lez-Beaupré $(310 \mathrm{~m})$ reçoivent un peu plus de précipitations que la station de Chevroz située à $250 \mathrm{~m}$ d'altitude (Bekkary, 1992).

Les stations de Roche-lez-Beaupré el Chevroz occupent une position topographique sommitale plane. La végétation est composée d'un taillis sous futaie dominé par le chêne (Quercus petraea et $Q$ robur) et le hêtre (Fagus silvatica), avec en sous-étage le charme (Carpinus betulus) et des arbustes comme le houx (llex aquifolium), le noisetier (Corylus avellana) et le chèvrefeuille (Lonicera periclymenum).

La station de Fontain correspond à un fond de vallon karstique. Elle comporte un taillis sous futaie en conversion avec des chênes pédonculés (Quercus robur) accompagnés par un sousétage de charme (Carpinus betulus), de frêne (Fraxinus excelsior), de noisetier (Corylus avellana) et d'érable champêtre (Acer campestre).

\section{Méthodes d'étude et caractéristiques des sols}

Pour décrire et prélever les horizons des sols, des excavations de $4 \times 2,5 \mathrm{~m}$ ont été creusées jusqu'à la roche. L'interface avec le banc calcaire a été atteinte entre 4 et $4,5 \mathrm{~m}$ à Fontain et entre 1,3 et $1,5 \mathrm{~m}$ à Roche-lez-beaupré. À Chevroz, la fosse a été ouverte au-delà de l'interface sol-alluvions sableuses $(1,6 \mathrm{~m})$ jusqu'à $3 \mathrm{~m}$. Les descriptions ont été effectuées selon le guide FAO (1977).

Sur les échantillons séchés à l'air et tamisés à $2 \mathrm{~mm}$, la texture a été déterminée par la méthode 
inernationale. Le $\mathrm{pH}$ (eau/sol $=2,5 / 1)$, la capacité d'échange cationique (méthode à l'acétate d'ammonium), les cations échangeables $\mathrm{Ca}, \mathrm{Mg}$, $\mathrm{K}$ (absorption atomique) et le carbone organique et minéral (dosage du $\mathrm{CO}_{2}$ au Carmographe Wösthoff) ont également été mesurés. La densité apparente a été mesurée en prélevant des volumes connus d'échantillons qui ont été séchés à $105^{\circ} \mathrm{C}$ et pesés. Cinq répétitions ont été réalisées pour chaque mesure. La porosité totale a été calculée à partir de la densité réelle $D r$ et de la densité apparente Da par la formule : $\mathrm{P}(\%)=$ $(1-D a / D r) \cdot 100$, avec $D r=2,65-0,015 . C(\%)$.

\section{Station de Roche-lez-Beaupré}

Le sol brun lessivé de Roche-lez-Beaupré est formé à partir du résidu d'altération des roches calcaires du Rauracien $\left(\mathrm{J}_{6}\right)$ et de limons éoliens (Gaiffe et Bruckert, 1985). II comprend des horizons organisés en unités structurales très cohérentes de petite taille, formées soit d'agrégats en grumeaux (horizons $A$ et $E$ ), soit d'agrégats polyédriques (horizons Bt). À l'interface sol-roche, la structure en polyèdres devient plus fine et les matériaux pédologiques pénètrent dans des cavités du banc calcaire sous-jacent.

La macroporosité est élevée dans l'ensemble du pédon et comporte des galeries fauniques (horizons $A$ et $E$, entre 0 et $40 \mathrm{~cm}$ ) et des vides planaires (horizons $\mathrm{Bt}_{1}-\mathrm{Bt}_{2}$ ). Les chenaux d'origine biologique ont une orientation dominante verticale. $\mathrm{A}$ partir de $\mathrm{EB}$ et en $\mathrm{Bt}_{1}-\mathrm{Bt}_{2}$, les vides sont toujours importants, mais résultent de processus physiques.

La densité apparente varie de $1,60 \mathrm{~g} . \mathrm{cm}^{-3}$ en EB à 1,79 en $\mathrm{Bt}_{2}$.

Du point de vue textural, c'est un sol limoneux qui présentent une forte augmentation de la teneur en argile avec la profondeur. II se caractérise par un $\mathrm{pH}$ acide (entre 4 et 4,9 ) et par des teneurs faibles en bases échangeables, sauf dans l'horizon profond où l'on note 13 m.éq. 100 $\mathrm{g}^{-1}$ de sol.

\section{Station de Chevroz}

Le sol lessivé glossique à fragipan de Chevroz est formé à partir d'un limon sableux alluvial qui constitue une terrasse du Riss (Bekkary, 1992). Les horizons $A$ et $E(0$ à $40 \mathrm{~cm})$ s'opposent fortement par leurs couleurs et leurs organisations aux horizons sous-jacents. Les premiers présentent une structure en agrégats d'origine biologique laissant place à une importante macroporosité. Les seconds constituent un matériau massif subdivisé en prismes de grandes dimensions, jointifs à leur base. Les glosses interpénètrent les horizons Btg sous forme d'un réseau polygonal large vers le haut, plus étroit vers le bas.

La densité apparente varie de $1,76 \mathrm{~g} \cdot \mathrm{cm}^{-3}$ en $E$ à 2,09 en Btg.

II s'agit d'un sol acide ( $\mathrm{pH}$ entre 4,4 et 4,9 ), désaturé, avec une teneur en $\mathrm{Ca}$ qui ne dépasse pas $0,7 \mathrm{~m}$.éq. $100 \mathrm{~g}^{-1}$.

\section{Station de Fontain}

La station de Fontain présente un sol brun lessivé colluvial profond installé sur un calcaire fissuré du Bathonien $\left(J_{2}\right)$. Les caractéristiques morphologiques observées dans les profils et les sections fines ont montré qu'à Fontain, les dépôts d'une épaisseur de $4 \mathrm{~m}$ qui recouvrent la roche calcaire karstifiée, sont fortement structurés en polyèdres et en agrégats. Tous les niveaux sont colorés de façon homogène en brun jaunâtre (10 YR) et brun rougeâtre (7,5 YR). Ils apparaissent soumis à un pédo-environnement aéré.

La densité apparente augmente régulièrement entre la surface $\left(0,87 \mathrm{~g} \cdot \mathrm{cm}^{-3}\right)$ et $100 \mathrm{~cm}(1,44$ g. $\left.\mathrm{cm}^{-3}\right)$; sa valeur moyenne pour les horizons situés au-dessous de $100 \mathrm{~cm}$ est de 1,49 $\pm 0,06$ g. $\mathrm{cm}^{-3}$. La CEC moyenne atteint 14,1 $\pm 2,7$ m.éq. $100 \mathrm{~g}^{-1}$.

Les horizons lessivés jusqu'à $60 \mathrm{~cm}$ sont partiellement désaturés et mésotrophes ; au-dessous de $60-80 \mathrm{~cm}$, le complexe d'échange devient saturé en bases. Le pH croît avec la profondeur, il est de 5,4 en surface, supérieur à 6,0 à $80 \mathrm{~cm}$ et dépasse 7,0 à $200 \mathrm{~cm}$. Le $\mathrm{pH}$ maximum est 7,7 à $420 \mathrm{~cm}$

\section{Mesures de I'humidité du sol}

Les mesures d'humidité du sol ont été réalisées par la méthode gravimétrique et grâce à des sondes capacitives.

Dans la méthode gravimétrique, les matériaux prélevés ( 3 répétitions par horizon et par station) 
sont pesés et séchés à l'étuve à $105^{\circ} \mathrm{C}$ (Vallée et Fédoroff, 1974 ; Blondé, 1989).

Les variations de l'humidité au cours de la journée ont été suivies à l'aide de sondes capacitives HUMICAP (Nardeux, 37552 Saint-Avertin) avec 2 répétitions par profondeur et par station.

Pour chaque station, 2 niveaux de profondeur ont été choisis en fonction de l'organisation des systèmes racinaires (Lucot et Bruckert, 1992) : le niveau de surface entre 35 et $45 \mathrm{~cm}$ et le niveau profond entre 80 et $100 \mathrm{~cm}$.

\section{Peuplements}

Les 3 stations sont traitées en taillis sous futaie avec une densité de peuplement moyenne (60 à 80 tiges par ha).

Les proportions entre les 2 espèces de chêne et leurs hybrides sont différentes selon la station, avec une dominance de chênes pédonculés à Fontain (95\%), alors que les chênes sessiles sont majoritaires à Chevroz $(55 \%)$ et à Roche-lezBeaupré (65\%).

L'âge moyen des arbres est de 140 ans à Fontain et de 120 ans à Chevroz et à Roche-lezBeaupré. Leur diamètre moyen est de $65 \mathrm{~cm}$ à Fontain, de $50 \mathrm{~cm}$ à Chevroz et de $55 \mathrm{~cm}$ à Roche-lez-Beaupré. Leur hauteur moyenne est de $24 \mathrm{~m}$ à Fontain, de $23 \mathrm{~m}$ à Chevroz et de $22 \mathrm{~m}$ à Roche-lez-Beaupré.

\section{Systèmes racinaires}

Dans chaque station, une fosse est creusée au pied de 2 chênes adultes, à $1 \mathrm{~m}$ de distance du tronc (Lucot et Bruckert, 1992). Sur un profil lissé en arc de cercle, on mesure la densité racinaire par tranche de $10 \mathrm{~cm}$ de profondeur. La densité est exprimée en pourcentage de la surface de profil occupé par les sections de racines. Les racines subverticales sont dégagées sous l'arbre. Leur profondeur de ramification ainsi que la profondeur maximum prospectée sont mesurées.

Le système racinaire de surface colonise le sol de manière intensive dans un rayon moyen de $3 \mathrm{~m}$ autour de l'arbre (Lucot et Bruckert, 1992). Ce rayon est de $2,5 \mathrm{~m}$ pour le système profond. II permet le calcul du volume de sol théoriquement prospectable par chaque partie du système racinaire.

\section{Potentiel hydrique foliaire}

Dans chacune des 3 stations, 15 chênes adultes et sains ont été choisis. Les prélèvements de rameaux ont été effectués à une hauteur comprise entre 2 et $3 \mathrm{~m}$, toujours sur la même branche. Des expériences préliminaires ont montré que le potentiel hydrique mesuré sur les branches basses est identique à celui mesuré au niveau du houppier. Pour des conditions constantes, le principal facteur de variation du potentiel hydrique est la position par rapport au soleil. Pour un déficit hydrique moyen, l'écart type de mesures de potentiel répétées sur une même branche est de 0,9 bar et de 1,2 bars sur des branches différentes.

Les organes prélevés sont lignifiés et longs de $15 \mathrm{~cm}$. Le potentiel hydrique est mesuré à l'aide d'une chambre de pression (Scholander et al, 1965) dans les 3 min suivant le prélèvement. Les mesures ont été effectuées durant les mois de juillet, août et septembre 1992. Dans chaque station, 4 séries de mesures journalières ont été réalisées, avec 6 mesures par jour sur 15 arbres (soit un total de 1080 mesures de potentiel hydrique foliaire).

Les 4 journées de mesure correspondent à une journée dans des conditions de sol humide, une journée de ressuyage du sol, une journée sèche et une journée d'humectation.

\section{Conditions météorologiques}

Les mesures ont été réalisées au cours de journées d'été sans vent et avec un ensoleillement moyen. La température et l'humidité relative sont enregistrées en continu au centre de la station à l'aide d'un thermohygromètre graphique. Le potentiel atmosphérique ( $\Psi$, bar) est calculé à partir de la formule:

$$
\Psi=\frac{8,314 \times(T+273)}{18} \times \operatorname{Ln} \frac{H r}{100} \times 10
$$

où $\mathrm{T}=$ température en ${ }^{\circ} \mathrm{C}, \mathrm{Hr}=$ humidité relative (\%).

\section{Étude statistique}

L'homogénéité des 15 valeurs de potentiel hydrique du xylème a été vérifiée à chaque 
heure de mesure par le test de Student. Les relations entre les paramètres sont calculées par régression linéaire multiple pas à pas, avec $P=0,05$ pour le test $F$. Cette méthode statistique permet de sélectionner et hiérarchiser la contribution de chaque facteur explicatif indépendant (Gouet et Philippeau, 1988). La linéarité des relations calculées est vérifiée graphiquement et par le test $F$

\section{RÉSULTATS}

\section{Humidité du sol}

Au cours d'une journée, en l'absence de précipitations, l'humidité du sol ne présente pas de variation significative. En revanche, d'une journée de mesure à l'autre, I'humidité volumique varie entre 15,6 et $37,9 \%$ en surface et entre 25,0 et $39,0 \%$ en profondeur.

Les 2 premières séries de mesures effectuées fin juin et mi-juillet donnent, selon la station, des valeurs de 28,4 et $37,9 \%$ en surface et de 25,0 à $38,2 \%$ en profondeur. La troisième série de mesures réalisée fin août présente les valeurs d'humidité les plus faibles : de 15,6 à $26,3 \%$ en surface et de 25,0 à $37,0 \%$ en profondeur. Des orages ont entraîné une réhumectation du sol lors de la quatrième série, avec des valeurs d'humidité proches de celles des 2 premières journées.

Globalement, la station de Fontain est la plus humide avec des valeurs moyennes pour les 2 niveaux de profondeur de 30,9 et $36,3 \%$. Les 2 autres stations possèdent des valeurs très proches d'environ $27 \%$ en surface et $30 \%$ en profondeur.

\section{Potentiel hydrique foliaire}

L'homogénéité des séries de 15 données pour chaque heure de mesure est vérifiée à $P=0,01$, ce qui signifie que les différences entre les valeurs de potentiel des 15 arbres ne sont pas significatives.

Des exemples des variations du potentiel hydrique moyen en fonction de l'heure de la journée dans les 3 stations sont donnés dans la figure 1. Quelle que soit la station considérée, le potentiel hydrique varie au cours de la journée depuis un minimum au point du jour jusqu'au maximum observé entre 12 et $15 \mathrm{~h}$ (fig 1). Au crépuscule, le potentiel a retrouvé un niveau proche de celui du matin.

L'analyse graphique des données et les approches statistiques descriptives classiques (comparaisons de moyennes) ne permettent pas de préciser les relations entre le potentiel hydrique foliaire et les autres paramètres mesurés. C'est pourquoi, des régressions multiples pas à pas ont été calculées, pour chaque station et pour l'ensemble des valeurs mesurées, entre le potentiel hydique des feuilles (variable dépendante) et les 3 variables explicatives supposées que sont le potentiel atmosphérique, I'humidité volumique du sol en surface et en profondeur. Cette méthode permet de hiérarchiser la contribution de chaque régresseur à l'explication des variations du facteur dépendant (potentiel hydrique foliaire). L'indépendance des variables a été testée et la linéarité des relations a été vérifiée statistiquement et graphiquement. Les coefficients de détermination des variables entrées dans la régression sont très significatifs : $P=0,001$ (tableau I).

Deux facteurs interviennent dans la régression pour les données de Roche-lezBeaupré et Chevroz, avec respectivement 90 et $71 \%$ de la variation du potentiel hydrique qui est expliquée. Dans les 2 cas, le paramètre explicatif le plus important est le potentiel atmosphérique. II explique à lui seul $83 \%$ de la variation à Roche-lez-Beaupré et $60 \%$ à Chevroz. Le deuxième facteur entré dans la régression est le paramètre humidité du sol à $50 \mathrm{~cm}$ pour la première 

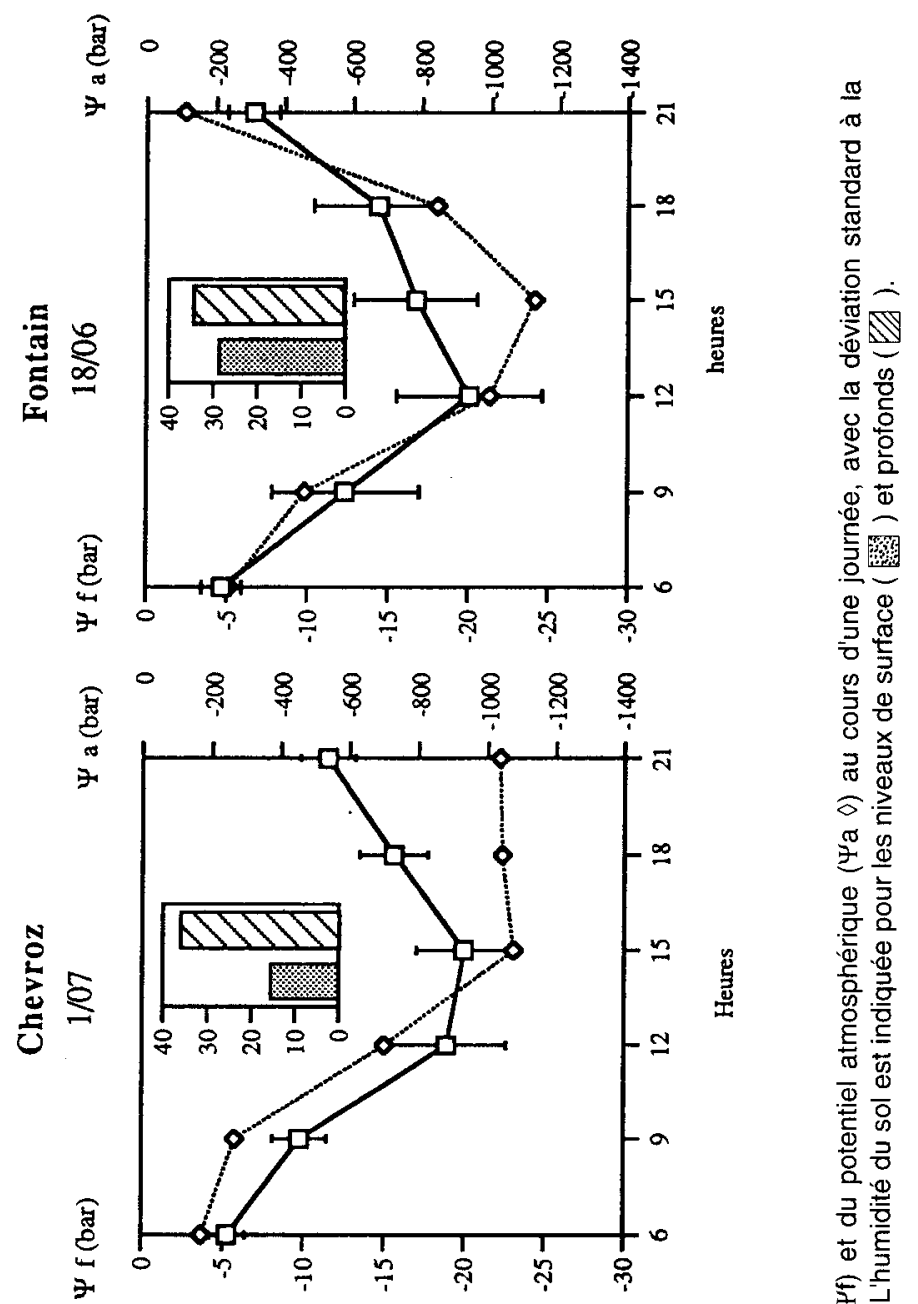

舫

응

ํํ용

o.

क

文

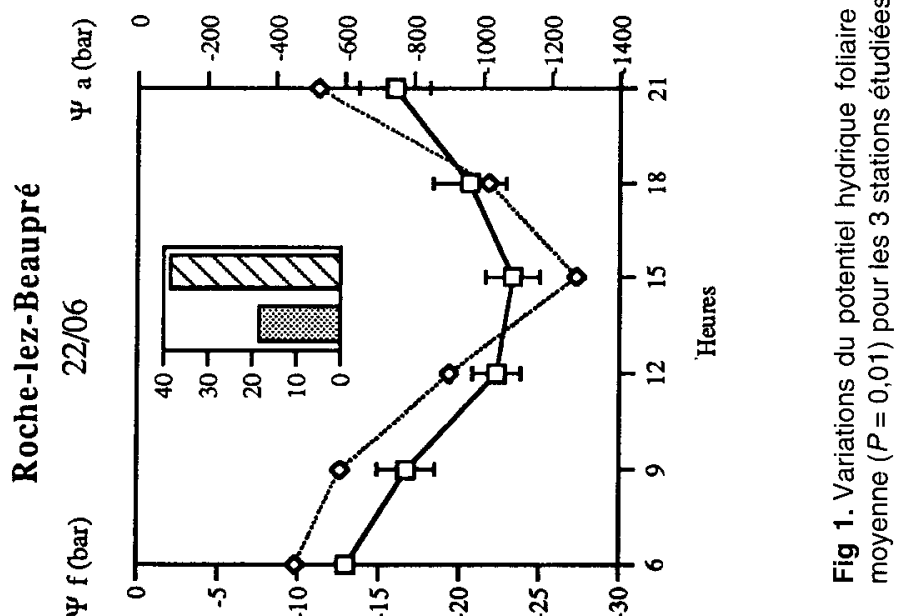


Tableau I. Coefficients de détermination $\left(\mathrm{r}^{2}\right)$ des 2 premiers pas des corrélations multiples effectuées pour 3 stations, 4 j par station et 6 mesures par jour.

Premier pas

Station

Roche-lez-Beaupré

Chevroz

Fontain

Facteur $n^{\circ} 1 \quad r^{2}$

Signification

Facteur $n^{\circ}$

Deuxième pas

\begin{tabular}{|c|c|c|c|c|c|c|}
\hline Station & Facteur $n^{\circ} 1$ & $r^{2}$ & Signification & Facteur $n^{\circ} 2$ & $r^{2}$ & Signification \\
\hline Roche-lez-Beaupré & $\Psi \mathrm{atm}$ & 0,83 & $\star \star \star \star$ & $\mathrm{Hs}$ & 0.90 & $\star \star \star *$ \\
\hline Chevroz & $\Psi$ atm & 0,60 & $\star \star \star$ & $\mathrm{Hp}$ & 0.71 & $\star \star \star$ \\
\hline Fontain & $\mathrm{Hp}$ & 0,58 & $\star \star \star *$ & - & - & - \\
\hline
\end{tabular}

$\mathrm{Hp}$ : humidité du sol pour les niveaux profonds.

station et le paramètre humidité du sol à $100 \mathrm{~cm}$ pour la seconde.

Un seul facteur explicatif est introduit de manière significative dans la régression pour les données de Fontain : I'humidité du sol à $100 \mathrm{~cm}$. II n'explique que $58 \%$ de la variation du potentiel hydrique des feuilles.

\section{Systèmes racinaires}

L'organisation des matériaux pédologiques des 3 stations se traduit par des paramètres racinaires différents (tableau II). À Rochelez-Beaupré, le système racinaire de surface colonise le sol jusqu'à $40 \mathrm{~cm}$ de profondeur et le maximum de densité racinaire est atteint entre 10 et $20 \mathrm{~cm}$ de profondeur. Les pivots se ramifient au niveau du maximum de densité, c'est-à-dire entre 40 et 50 $\mathrm{cm}$. Les ramifications des pivots ont des directions très changeantes et parviennent à $1,5 \mathrm{~m}$ de profondeur, entre les éléments calcaires. L'enracinement intensif dispose seulement de $14 \mathrm{~m}^{3}$ pour se développer.

À Chevroz, le système de surface s'étend jusqu'à une profondeur de $40 \mathrm{~cm}$, avec un maximum de densité racinaire entre 25 et $35 \mathrm{~cm}$ de profondeur. La profondeur de ramification moyenne des pivots est observée entre 60 et $70 \mathrm{~cm}$. Certaines des racines issues de ces ramifications parviennent à s'insérer dans les glosses, puis elles prennent une direction verticale et on les retrouve jusqu'à une profondeur de $3 \mathrm{~m}$. Les racines du système intensif peuvent avoir accès à un volume de $18 \mathrm{~m}^{3}$.

À Fontain, les racines subhorizontales du système de surface prospectent le sol jusqu'à $60 \mathrm{~cm}$ de profondeur avec un maximum de densité racinaire entre 20 et $30 \mathrm{~cm}$ de profondeur. Les racines subverticales (pivots) du système racinaire extensif profond parviennent à plus de $4 \mathrm{~m}$ de profondeur. Ces pivots se ramifient très peu et lorsque la ramification existe, elle se produit entre 90 et $100 \mathrm{~cm}$ de profondeur. Le volume de sol prospectable pour l'enracinement intensif est estimé à $23 \mathrm{~m}^{3}$.

\section{DISCUSSION, CONCLUSION}

Les variations du potentiel hydrique du xylème sont liées d'une part aux conditions atmosphériques qui modulent la transpiration et d'autre part aux conditions pédologiques dont dépend la disponibilité en eau (Hillel, 1974 ; Aussenac, 1985 ; Crombie et al, 1988 ; Carlier et al, 1992).

Précédemment, nous avons montré que ces différents facteurs de variation du poten- 
Tableau II. Valeurs moyennes des paramètres racinaires mesurés dans les 3 stations. Le volume de sol prospectable correspond au volume que les racines peuvent théoriquement coloniser de manière intensive.

Paramètres racinaires

Système de surface

Maximum de densité $(\mathrm{cm})$ Profondeur maximale $(\mathrm{cm})$

Volume prospectable $\left(\mathrm{m}^{3}\right)$

Système profond

Profondeur de ramification $(\mathrm{cm})$

Profondeur maximale $(\mathrm{cm})$

Volume prospectable $\left(\mathrm{m}^{3}\right)$

Volume total de sol prospectable $\left(\mathrm{m}^{3}\right)$
Roche-lez-Beaupré Chevroz

Fontain

$25-35$
40
12

60-70

300

6

18

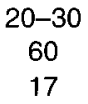

17

$90-100$

$>450$

6

23 tiel hydrique interviennent avec des poids différents selon le moment de la journée considéré (Badot et al, 1994). Dans le présent travail, nous avons cherché à mettre en évidence l'influence relative du potentiel hydrique atmosphérique, de l'humidité du sol en surface et en profondeur sur les variations du potentiel hydrique en fonction des caractéristiques pédologiques des stations forestières étudiées. À Roche-lez-Beaupré et à Chevroz, c'est le potentiel atmosphérique qui constitue le facteur principal de variation du potentiel hydrique, alors qu'à Fontain, c'est l'humidité du sol en profondeur qui rend compte de ces variations. Or les stations étudiées s'opposent par leurs propriétés pédologiques qui influent sur la quantité d'eau disponible. L'humidité du sol est donc susceptible d'expliquer les différences mises en évidence entre les 3 stations.

Pour une espèce d'arbre donnée, la disponibilité effective de l'eau du sol est d'abord dépendante de la répartition de ses racines (Crombie et al, 1988 ; Callaway, 1990). La distribution des racines des systèmes de surface et de profondeur indique un volume rhizofonctionnel croissant de Roche-lezBeaupré à Chevroz et de Chevroz à Fontain. Les stations de Roche-lez-Beaupré et de Chevroz sont similaires pour leur système de surface, mais le système profond de la première station exploite une profondeur de moitié moins importante que celui de la seconde. Le sol de Fontain autorise un développement racinaire maximal sans contrainte (Lucot et Bruckert, 1992).

La mise en relation de ces caractéristiques racinaires avec les résultats des régressions montre que les variations du potentiel hydrique du xylème dépendent d'autant plus des conditions atmosphériques que le sol est contraignant pour le système racinaire. Ainsi, le potentiel atmosphérique explique $83 \%$ des variations du potentiel hydrique dans la station la plus contraignante, c'est-à-dire Roche-lez-Beaupré. À Fontain, les conditions sont peu contraignantes et le potentiel hydrique du xylème n'est pas lié aux variations du potentiel atmosphérique. La gamme des conditions climatiques et pédologiques non-extrêmes étudiées ici explique la linéarité des relations calculées. 
À Roche-lez-Beaupré, les racines se développent principalement en surface et c'est effectivement l'humidité du sol en surface qui constitue le deuxième facteur explicatif. À Chevroz, comme le laisse supposer la distribution des racines, c'est l'humidité en profondeur qui est le premier facteur explicatif. Dans le cas d'un développement racinaire optimal (Fontain), l'humidité du sol en profondeur représente le facteur principal de variation du potentiel hydrique du xylème.

Dans les 2 stations où le développement des racines n'est pas limité (Chevroz et Fontain), c'est l'humidité en profondeur qui régit en partie l'alimentation en eau de l'arbre. Le système racinaire profond, c'est-à-dire les niveaux inférieurs à $60 \mathrm{~cm}$, serait responsable de l'alimentation en eau dans les conditions de relative sécheresse observées pendant la campagne de mesures. La compartimentation architecturale du système racinaire aurait ainsi des répercussions sur le fonctionnement physiologique (Badot et al, 1994).

L'arbre a d'autant plus de difficultés à s'adapter à la sécheresse que le système racinaire colonise un volume de sol faible et que la quantité d'eau disponible est par conséquent limitée. Les caractéristiques pédologiques (granulométrie, structuration, porosité, perméabilité) différentes d'une station à l'autre ne sont pas directement prises en compte dans la régression, néanmoins les paramètres racinaires étudiés en sont tributaires et les reflètent.

Le sol lessivé glossique à fragipan (Chevroz), peu perméable, semble moins contraignant que le sol brun lessivé sur calcaire (Roche-lez-Beaupré) au niveau de la colonisation racinaire et de l'alimentation en eau. Dans le sol hydromorphe (Chevroz), la contrainte mécanique que constitue le niveau tassé pour la colonisation en profondeur est compensée par sa faible perméabilité. Celle-ci accroît l'eau disponible tant en quantité que dans la durée. À Fontain, le sol est perméable mais la capacité de rétention et le volume prospecté sont plus importants.

Selon certains travaux récents, les réactions de la plante face à un stress hydrique dépendent de l'espèce considérée (Aussenac, 1985 ; Crombie et al, 1988). Des différences existent entre les exigences autécologiques du chêne pédonculé et du chêne sessile (Rameau et al, 1989). Les peuplements étudiés ici sont composés presque exclusivement de chênes pédonculés (Fontain), ou d'un mélange de chêne pédonculés et sessiles avec leurs hybrides (Chevroz et Roche-lez-Beaupré). Or les échantillons observés donnent des séries de mesures très homogènes malgré le mélange des 2 espèces et leurs hybrides. Cette observation est en accord avec les résultats de Dreyer et al (1990) qui ont souligné les difficultés pour différencier les espèces de chênes par leurs réactions physiologiques. Ces auteurs ont montré d'une part l'absence de différence entre les capacités des tissus foliaires des chênes pédonculés et sessiles à tolérer un assèchement et d'autre part l'importance des capacités d'adaptation des individus en fonction des conditions stationnelles.

Des séries de mesures complémentaires seront nécessaires pour établir les relations entre les différents paramètres dans des situations de contraintes hydriques fortes, assèchement marqué ou stations installées sur calcaire superficiel avec des sols plus contraignants pour les systèmes racinaires. L'étude des relations entre les caractéristiques des sols, les capacités d'enracinement des différentes espèces et la disponibilité de l'eau devrait permettre de mieux cerner les critères à respecter lors du choix des espèces à maintenir ou à planter sur une station donnée. Ces informations pourraient aussi permettre de progresser dans la compréhension de certains phénomènes de dépérissement et de perte de vitalité qui affectent actuellement les écosystèmes forestiers de l'est de la France. 


\section{REMERCIEMENTS}

Ce travail a été réalisé grâce au soutien financier du ministère de la Recherche et de la Technologie (contrat université de FrancheComté/INRA/MRT $91 \mathrm{G}$ 0848).

Les auteurs remercient également les communes de Fontain, Roche-lez-Beaupré et Chevroz, ainsi que l'Office national des forêts pour leur collaboration.

\section{RÉFÉRENCES}

Aussenac G (1985) Le potentiel hydrique de l'arbre: une donnée essentielle pour la compréhension de l'écophysiologie des essences forestières. Sci Sol 4, 217-226

Aussenac G, Finkelstein D (1983) Influence de la sécheresse sur la croissance et la photosynthèse du cèdre. Ann Sci For 40 (1), 67-77

Aussenac $G$, Levy $G$ (1983) Influence du dessèchement du sol sur le comportement hydrique et la croissance du chêne pédonculé (Quercus pedonculata Ehrl) et du frêne (Fraxinus excelsior $L$ ) cultivés en case de végétation. Ann Sci For 40 (3), 251-264

Aussenac G, Granier A (1984) Influence du dessèchement du sol sur le fonctionnement hydrique et la croissance du Douglas (Pseudotsuga mensiesii (Mirb) Franco). Acta CEcologica/CEcol plant 5 (3), 241-253

Badot PM, Garrec JP (1990) Perturbation hydrique et altération des surfaces dans les aiguilles d'épicéas (Picea abies L) du Jura en fonction de leur âge et de l'état de dépérissement des arbres. Ann For Sci 21, 591-598

Badot PM, Lucot E, Bruckert S (1994) L'humidité du sol en profondeur constitue, en milieu de journée, la principale source de variation du potentiel hydrique foliaire de peuplements de chênes (Quercus sp). CR Acad Sci, Sciences de la Vie, 317, 341-345

Bekkary M (1992) Effet de la fracturation et de la porosité des roches sur l'organisation et les régimes hydriques et atmosphériques de sols à horizon argilique ou fragique. Thèse doct $3^{e}$ cycle, univ FrancheComté, Besançon, France, $199 \mathrm{p}$

Blondé JL (1989) Influence du régime hydrique induit par la fissuration des roches sur l'humification et l'organisation des sols en milieu calcique. Thèse doct $3^{e}$ cycle, univ Franche-Comté, Besançon, France, 145 p
Callaway RM (1990) Effects of soil water distribution on the lateral root development of 3 species of California oaks. Amer J Bot 77, 1469-1475

Carlier G, Peltier JP, Gielly L (1992) Comportement hydrique du frêne (Fraxinus excelsior $\mathrm{L}$ ) dans une formation montagnarde mésoxérophile. Ann Sci For $49,207-223$

Crombie DS, Tippett JT, Hill TC (1988) Dawn water potential and root depth of trees and understorey species in south-western Australia. Aust J Bot 36, $621-631$

Dreyer E, Colin-Belgrand M, Souiller I, Biron P, Bousquet $F$, Aussenac $G(1990)$ Diversité des caractéristiques écophysiologiques des chênes européens. Quelques exemples. Rev For Fran XLII 2, 174-181

FAO (1977) Guidelines for soil profile description. Food and agriculture organization of the United Nations, Rome, Italie, $125 \mathrm{p}$

Gaiffe M, Bruckert S (1985) Analyse des transports de matières et des processus pédogénétiques impliqués dans les chaînes de sols du karst jurassien. Catena ${ }^{\circ}$ spécial (Soils and Geomorphology) 6 , 159-174

Gouet JP, Philippeau G (1988) Comment interpréter les résultats d'une régression. SESI-ITCF, Paris, $55 \mathrm{p}$

Guillaumin JJ, Bernard C, Delatour C, Belgrand M (1983) Le dépérissement du chêne à Tronçais : pathologie racinaire. Rev For Fra 35 (6), 415-424

Hillel D (1974) L'eau et le sol, principes et processus physiques. Vander, Louvain, $257 \mathrm{p}$

Kessler J, Oosterbaan RJ (1974) Determining hydraulic conductivity of soils. In : Drainage principles and applications, 3, 252-296

Lucot E, Bruckert S (1992) Organisation du systeme racinaire du chêne pédonculé (Quercus robur) développé en conditions édaphiques non contraignantes (sol brun lessivé colluvial). Ann Sci For $49,465-479$

Rameau JC, Mansion D, Dume G (1989) Flore forestière française. I. Plantes et collines. IDF, Paris, $1784 \mathrm{p}$

Saugier B, Halledin S, Pontailler JY, Nizinski G (1985) Bilan hydrique de forêts de chêne et de hêtre à Fontainebleau. Mesures et modélisation. Revue du Palais de la Découverte 13,130,187-200

Scholander PF, Hammel HT, Bradstreet ED, Hemmingsen EA (1965) Sap pressure in vascular plants. Science 148, 339-346

Vallée C, Fédoroff A (1974) Comportement hydrique d'un sol d'altération de granite sous une pessière. Sci Sol 3, 119-131 